\title{
Distribution of Stochastic Impulses Acting on an Oscillator as a Function of Its Motion
}

\author{
M. JABŁOŃSKI ${ }^{a}$ AND A. OzGA ${ }^{b}$ \\ ${ }^{a}$ Faculty of Mathematics and Computer Science, Jagiellonian University \\ Gołębia 24, 31-007 Cracow, Poland \\ ${ }^{b}$ Department of Mechanic and Vibroacoustics, University of Science and Technology \\ al. Mickiewicza 30, 30-059 Cracow, Poland
}

\begin{abstract}
In previous papers formulas have been derived describing distribution of a random variable whose values are positions of an oscillator at the moment $t$, which, in the interval $[0, t]$, underwent the influence of stochastic impulses with a given distribution. In this paper we present reasoning leading to an opposite inference thanks to which, knowing the course of the oscillator, we can find the approximation of distribution of stochastic impulses acting on it. It turns out that in the case of an oscillator with damping the stochastic process $\xi_{t}$ of its deviations at the moment $t$ is a stationary and ergodic process for large $t$. Thanks to this, time average of almost every trajectory of the process, which is the $n$-th power of $\xi_{t}$ is very close to the mean value of $\xi_{t}^{n}$ in space for sufficiently large $t$. Thus, having a course of a real oscillator and theoretical formulae for the characteristic function $\xi_{t}$ we are able to calculate the approximate distribution of stochastic impulses forcing the oscillator.
\end{abstract}

PACS numbers: 45.10.-b, $45.30+\mathrm{s}$

\section{Introduction}

In the papers $[1,2]$ we considered mechanical systems such as oscillator and string and analyzed their response to impulses whose magnitude as well as the place and moment of action were random. There we derived theoretical formulas regarding the mean value and variance of positions of the point of a given mechanical system as a function of statistical properties of these impulses. It turns out that statistical behavior of a mechanical system with damping or without damping is the same as the behavior of the mechanical system subjected to action of continuous forces equal to the mean value of impulses. The derived formulas are consistent with computer simulations. However, in practical applications, for example in control of a technological process, we need inverse dependence, that is, we need distributions of stochastic impulses as a function of the motion of a mechanical system. In this paper we try to solve this problem.

We should remember that the stochastic process $\left\{\xi_{t}^{n}, t \in R^{+}\right\}$is stationary if for any $k$, any open subsets $A_{1}, A_{2}, \ldots A_{k}$ of $\mathrm{R}$ and for any $t_{1}<t_{2}<\ldots<t_{k}$ the probability $P\left(\xi_{t_{1}+h}^{-1}\left(A_{1}\right), \xi_{t_{1}+h}^{-1}\left(A_{2}\right), \ldots, \xi_{t_{k}+h}^{-1}\left(A_{k}\right)\right)$ does not depend on $h$. The stationary and stochastic process is ergodic if for almost all $\omega$ in $\Omega \lim _{t \rightarrow \infty} \frac{1}{t} \int_{0}^{t} \xi_{s}(\omega) \mathrm{d} s=$ $\int_{0}^{t} \xi_{0}(\omega) \mathrm{d} \omega$ (the mean value over the space is the mean value over time).

The previous papers suggest that we need to take large probes of trajectories which start from positions equal to zero to find statistical moments of considered motion. In practice it is impossible to achieve. Fortunately, it turns out that in the case of an oscillator with damping its behavior tends to be an ergodic and stationary process. Therefore we can take one sufficiently long trajectory of the motion of this oscillator and get all the statistical moments of the position of the considered oscillator at moment $t$ for large enough $t$. The statistical moments, in turn, give us an approximation of distribution of impulses, which allows us to control a technological process or adjust the parameters of the oscillator. The formulas for distributions are confirmed by computer simulations.

The first partial mathematical results regarding vibration of oscillators forced by stochastic impulses can be found in the following works [3-15]. Monographs [1618] introduce the foundations of the considered problems. Works $[4,5]$ include certain results concerning nonlinear systems subjected to stochastic forces that might not act in a continuous way.

\section{Main results}

Let us consider the differential equation of the forced harmonic oscillator with damping

$$
\frac{\mathrm{d}^{2} x}{\mathrm{~d} t^{2}}+2 b \frac{\mathrm{d} x}{\mathrm{~d} t}+a^{2} x=f(t)
$$

where $0<b<a$. The solution of the above equation satisfying the following initial condition

$$
x(0)=0
$$

and

$$
\dot{x}(0)=0
$$

has the form 


$$
x(t)=\frac{1}{\sqrt{a^{2}-b^{2}}} \int_{0}^{t} \frac{f(u) \sin \left(\sqrt{a^{2}-b^{2}}(t-u)\right)}{\exp (b(t-u))} \mathrm{d} u .(4)
$$

If $\eta_{i}$ is any sequence of real numbers, $t_{i}$ is any increasing sequence of real numbers and $f(t)$ is given by

$$
f(t)=\sum_{t_{i}<t} \eta_{i} \delta_{t_{i}}
$$

where $\delta_{t_{i}}$ are $\delta$-Dirac distributions at $t_{i}$, then the solution of (1), (2) (3) takes the following form

$$
\begin{aligned}
x(t) & =\frac{1}{\sqrt{a^{2}-b^{2}}} \\
\times & \sum_{t_{i}<t} \eta_{i} \exp \left(-b\left(t-t_{i}\right)\right) \sin \left(\sqrt{a^{2}-b^{2}}\left(t-t_{i}\right)\right) .
\end{aligned}
$$

Let $\eta_{i}$ be independent and identically distributed random variables with finite mean value and let $\tau_{i}=t_{i}-t_{i-1}$, $i=1,2, \ldots$, be independent and identically distributed random variables with exponential distribution $F(u)=$ $1-\exp (-\lambda u)$ for $u>0$ and $F(u)=0$ for $u<0$ and for some $\lambda>0$. Applying to (1) Theorem 1 from [5] with $m=1, h_{1}=1$ and function

$$
g=\frac{1}{\sqrt{a^{2}-b^{2}}} \frac{\sin \left(\sqrt{a^{2}-b^{2}} t\right)}{\exp (b t)},
$$

we get the characteristic function of random variable (6)

$$
\begin{aligned}
& \varphi(s)=\exp \left(\lambda t \left[\int_{0}^{\infty} \int_{0}^{1} \exp \left(\frac{\mathrm{i} s y\left(\sin \sqrt{a^{2}-b^{2}} t u\right)}{\sqrt{a^{2}-b^{2}} \exp (b t)}\right)\right.\right. \\
& \left.\left.\mathrm{d} u \phi_{\zeta}(\mathrm{d} y)-1\right]\right) .
\end{aligned}
$$

This function allows us to find stochastic moments of random variable $\mathrm{x}(\mathrm{t})$ of any rate from the following formula

$$
m_{n}(t)=E\left(x^{n}(t)\right)=\frac{\varphi_{t}^{(n)}(0)}{i^{n}} .
$$

Now we derive formulas for $n$-th derivative of $\varphi_{t}(s)$. The first derivative has the form

$$
\begin{aligned}
& \varphi_{t}^{\prime}(s)=\varphi_{t}(s) \lambda t \int_{0}^{\infty} \int_{0}^{1}\left(\frac{\mathrm{i} y \sin \sqrt{a^{2}-b^{2}} t u}{\sqrt{a^{2}-b^{2}} \exp (b t u)}\right) \\
& \quad \times \exp \left(\frac{\mathrm{i} s y \sin \sqrt{a^{2}-b^{2}} t u}{\sqrt{a^{2}-b^{2}} \exp (b t u)}\right) \mathrm{d} u \phi_{\eta}(\mathrm{d} y) .
\end{aligned}
$$

Knowing that

$$
h^{(n)}=\left(h_{1} h_{2}\right)^{(n)}=\sum_{i=0}^{n}\left(\begin{array}{c}
n \\
i
\end{array}\right) h_{1}^{(n-i)} h_{2}^{(i)}
$$

and setting

$$
h_{1 t}(s)=\varphi_{t}(s)
$$

and

$$
\begin{aligned}
& h_{2 t}(s)=\lambda t \int_{0}^{\infty} \int_{0}^{1}\left(\frac{\mathrm{i} y \sin \sqrt{a^{2}-b^{2}} t u}{\sqrt{a^{2}-b^{2}} \exp (b t u)}\right) \\
& \quad \times \exp \left(\frac{\mathrm{i} s y \sin \sqrt{a^{2}-b^{2}} t u}{\sqrt{a^{2}-b^{2}} \exp (b t u)}\right) \mathrm{d} u \phi_{\eta}(\mathrm{d} y),
\end{aligned}
$$

$$
\begin{aligned}
& \left(\varphi_{t}^{\prime}(s)\right)^{(n)}=\sum_{j=0}^{n}\left(\begin{array}{c}
n \\
j
\end{array}\right)\left(\varphi_{t}(s)\right)^{(n-j)} \lambda t \\
& \quad \times \int_{0}^{\infty} \int_{0}^{1}\left(\frac{i^{(j+1)} y^{(j+1)} \sin ^{(j+1)} \sqrt{a^{2}-b^{2}} t u}{\left(\sqrt{a^{2}-b^{2}}\right)}\right) \\
& \quad \times \exp \left(\frac{\mathrm{i} s y \sin \sqrt{a^{2}-b^{2}} t u}{\sqrt{a^{2}-b^{2}} \exp (b t u)}\right) \mathrm{d} u \phi_{\eta}(\mathrm{d} y) .
\end{aligned}
$$

Setting in the last formula $s=0$, and integrating with respect to $y$ we obtain

$$
\begin{aligned}
& \left(\varphi_{t}^{\prime}(0)\right)^{(n)}=\sum_{j=0}^{n}\left(\begin{array}{c}
n \\
j
\end{array}\right)\left(\varphi_{t}(0)\right)^{(n-j)} \frac{\lambda t i^{(j+1)} E\left(\eta^{(j+1)}\right)}{\left(\sqrt{a^{2}-b^{2}}\right)^{(j+1)}} \\
& \times \int_{0}^{1}\left(\frac{\sin ^{(j+1)} \sqrt{a^{2}-b^{2}} t u}{\exp ((j+1) b t u)}\right) \mathrm{d} u .
\end{aligned}
$$

Dividing the last formula by sides by $i^{(j+1)}$ we get

$$
\begin{aligned}
& m_{n+1}(t)=\sum_{j=0}^{n}\left(\begin{array}{l}
n \\
j
\end{array}\right) m_{n-j}(t) \frac{\lambda t E\left(\eta^{(j+1)}\right)}{\left(\sqrt{a^{2}-b^{2}}\right)^{(j+1)}} \\
& \times \int_{0}^{1}\left(\frac{\sin ^{(j+1)} \sqrt{a^{2}-b^{2}} t u}{\exp ((j+1) b t u)}\right) \mathrm{d} u .
\end{aligned}
$$

Substituting $\sqrt{a^{2}-b^{2}} t u=v$ in (16) we obtain

$$
\begin{aligned}
& m_{n+1}(t)=\sum_{j=0}^{n}\left(\begin{array}{l}
n \\
j
\end{array}\right) m_{n-j}(t) \frac{\lambda t E\left(\eta^{(j+1)}\right)}{\left(\sqrt{a^{2}-b^{2}}\right)^{(j+1)}} \\
& \times \int_{0}^{\sqrt{a^{2}-b^{2}} t}\left(\frac{\sin ^{(j+1)} v}{\exp \left((j+1) b v / \sqrt{a^{2}-b^{2}}\right)}\right) \mathrm{d} v .
\end{aligned}
$$

Let us denote for brevity

$$
\begin{aligned}
& c=\sqrt{a^{2}-b^{2}}, \\
& \psi_{j r}(t)=\int_{0}^{c t} \frac{\sin ^{r} v}{\exp (j b v / c)} \mathrm{d} v
\end{aligned}
$$

and

$$
\psi_{j}=\psi_{j j} .
$$

We show that there is $\lim _{t \rightarrow \infty} \psi_{j}$. To do this we use the following formulae

$$
\begin{aligned}
& \int \mathrm{e}^{\beta x} \sin ^{r}(x) \mathrm{d} x=\frac{\mathrm{e}^{\beta x} \sin ^{r-1}(x)}{\beta^{2}+r^{2}}(\beta \sin (x)-r \cos (x)) \\
& +\frac{r(r-1)}{\beta^{2}+r^{2}} \int \mathrm{e}^{\beta x} \sin ^{r-2}(x) \mathrm{d} x, \\
& \int \mathrm{e}^{\beta x} \mathrm{~d} x=\frac{\mathrm{e}^{\beta x}}{\beta}, \\
& \int \mathrm{e}^{\beta x} \sin (x) \mathrm{d} x=\frac{\mathrm{e}^{\beta x}}{\beta^{2}+r^{2}}(\beta \sin (x)-\cos (x)),
\end{aligned}
$$

for any $j(22)$ and (23) imply respectively

$$
\lim _{t \rightarrow \infty} \psi_{j 1}(t)=\frac{1}{\left(\frac{j b}{c}\right)^{2}+1}
$$

we get 


$$
\lim _{t \rightarrow \infty} \psi_{j 0}(t)=\frac{c}{j b}
$$

Moreover, from (21) for $j>1$ and $r>1$ we get

$$
\begin{gathered}
\lim _{t \rightarrow \infty} \psi_{j r}(t)=\frac{\sin ^{(r-1)} v}{\exp (j b v / c)\left((j b / c)^{2}+r^{2}\right)} \\
\quad \times\left.\left(\left(\frac{-j b}{c}\right)^{2} \sin (v)-r \cos (v)\right)\right|_{0} ^{c t} \\
+\frac{r(r-1)}{(j b / c)^{2}+r^{2}} \int_{0}^{c t} \frac{\sin ^{(r-2)} v}{\exp (j b v / c)} \mathrm{d} v .
\end{gathered}
$$

Equality (26) for $r>1$ and $j>1$ implies

$$
\lim _{t \rightarrow \infty} \psi_{j r}(t)=\frac{r(r-1)}{(j b / c)^{2}+r^{2}} \lim _{t \rightarrow \infty} \psi_{j(r-2)}(t) .
$$

From $(24),(25)$ and (27) for $j$ even and $j>1$ we obtain

$$
\begin{aligned}
\lim _{t \rightarrow \infty} \psi_{j}(t)=\frac{j !}{\prod_{r=0}^{j / 2-1}\left((j b / c)^{2}+(j-2 r)^{2}\right)} \lim _{t \rightarrow \infty} \psi_{j 0}(t) \\
=\frac{j !}{\prod_{r=0}^{j / 2-1}\left((j b / c)^{2}+(2 r)^{2}\right)} \frac{c}{j b},
\end{aligned}
$$

whereas for odd $j$

$$
\begin{aligned}
& \lim _{t \rightarrow \infty} \psi_{j}(t) \\
& \quad=\frac{j !}{\prod_{r=0}^{(j-1) / 2-1}\left((j b / c)^{2}+(j-2 r)^{2}\right)} \lim _{t \rightarrow \infty} \psi_{j 1}(t) \\
& \quad=\frac{j !}{\prod_{r=0}^{(j-1) / 2-1}\left((j b / c)^{2}+(2 r+1)^{2}\right)}
\end{aligned}
$$

Knowing that

$$
m_{0}=\lim _{t \rightarrow \infty} \varphi_{t}(0)=1,
$$

using (17), (28) and (29) we can prove by induction that for any $n \geq 0$ there is limit

$$
\lim _{t \rightarrow \infty} \frac{\varphi_{t}^{(n)}(0)}{i^{n}}=\lim _{t \rightarrow \infty} m_{n}(t)=m_{n} .
$$

Moreover, for $n \geq 0$ we obtain the following equations

$$
m_{n+1}=\sum_{j=0}^{n}\left(\begin{array}{l}
n \\
j
\end{array}\right) m_{(n-j)} \frac{\lambda E\left(\eta^{(j+1)}\right)}{c^{2+j}} C(j+1),
$$

where $C(j)=\lim _{t \rightarrow \infty} \psi_{j}(t)$ for $j \geq 0$.

By (32) for $n=0$ we have

$$
m_{1}=m_{0} \frac{\lambda E(\eta) C(1)}{c^{2}}=\frac{\lambda E(\eta) C(1)}{c^{2}} .
$$

Hence

$$
\lambda=\frac{m_{1} c^{2}}{E(\eta) C(1)} .
$$

Substituting this for $\lambda$ in the formula (32) we get for any $n>0$

$$
\begin{aligned}
& m_{n+1}=\sum_{j=0}^{n}\left(\begin{array}{l}
n \\
j
\end{array}\right) m_{(n-j)} \frac{E\left(\eta^{(j+1)}\right)}{c^{2+j}} \\
& \times C(j+1) \frac{m_{1} c^{2}}{C(1) E(\eta)} .
\end{aligned}
$$

Multiplying the above equation by $E(\eta)$ by sides we get

$$
\begin{aligned}
& \sum_{j=0}^{n}\left(\begin{array}{l}
n \\
j
\end{array}\right) m_{(n-j)} m_{1} E\left(\eta^{(j+1)}\right) \frac{C(j+1)}{C(1) c^{j}} \\
& \quad-m_{n+1} E(\eta)=0 .
\end{aligned}
$$

Now we can write

$$
\begin{aligned}
& \left(m_{n} m_{1}-m_{n+1}\right) E(\eta) \\
& \quad+\sum_{j=1}^{n}\left(\begin{array}{l}
n \\
j
\end{array}\right) m_{(n-j)} m_{1} E\left(\eta^{(j+1)}\right) \frac{C(j+1)}{C(1) c^{j}}=0 .
\end{aligned}
$$

If random variable $\eta$ assumes a finite number of known values $\left\{x_{1}, x_{2}, \ldots, x_{k}\right\}$ with unknown probabilities $p_{j}=$ $P\left(\eta=x_{j}\right)$ for $n=1,2, \ldots, k$, then the Eq. (37) for $0<n<k$ with obvious equality

$$
m_{0}=1=\sum_{j=1}^{k} p_{j}
$$

allows us to find distribution $p_{j}$ when $m_{n}$ are known. Since $E(\eta)=\sum_{i=1}^{k} \eta_{i} p_{i}$ Eq. (37) is equivalent to

$$
\begin{aligned}
& \sum_{i=1}^{k} p_{i}\left[\left(m_{n} m_{1}-m_{n+1}\right) \eta_{i}\right. \\
& \left.\quad+\sum_{j=1}^{n}\left(\begin{array}{l}
n \\
j
\end{array}\right) m_{(n-j)} m_{1} \eta^{(j+1)} \frac{C(j+1)}{C(1) c^{j}}\right]=0 .
\end{aligned}
$$

Set

$$
\begin{aligned}
a_{n i} & =\left(m_{n} m_{1}-m_{n+1}\right) \eta_{i} \\
& +\sum_{j=1}^{n}\left(\begin{array}{c}
n \\
j
\end{array}\right) m_{(n-j)} m_{1} \eta^{(j+1)} \frac{C(j+1)}{C(1) c^{j}},
\end{aligned}
$$

for $n=1,2, \ldots, k-1$ and $i=1,2, \ldots, k$ and

$$
a_{k i}=1 \text {, }
$$

for $i=1,2, \ldots \mathrm{k}$.

To find $p_{i}$ it is enough now to solve the following equation

$$
A P=Y
$$

where $Y=(0,0, \ldots, 0,1)$ and $P=\left(p_{1}, p_{2}, \ldots, p_{k}\right)$.

In practice we can calculate values $m_{k}$ taking the mean value of the $k$-th powers of the measured oscillator's positions and using ergodicity of the process $x(t)$ that is using the formula

$$
E(x(t)) \cong \frac{1}{k} \sum_{i=1}^{k} x\left(\frac{t i}{k}\right)
$$

which is valid for large $t$ and $k$.

If we add to Eq. (37)two additional equations for $n=k$ and $n=k+1$ then we can also calculate parameters $a$ and $b$ of the oscillator. However, this last problem is a complicated task since coefficients in Eq. (32) are polinomials with respect to $a$ and $b$ of a very high degree.

In many cases random variable $\eta$ is a continuous one. If it is so we can find aproximation of the distributtion of $\eta$ whenever $\eta$ is bounded. It is enough to take numbers $x_{1}, x_{2}, \ldots, x_{k}$ uniformly displayed in the interval of the values assumed by $\eta$ and find probabilities corresponding to $x_{1}, x_{2}, \ldots, x_{k}$ by $(42)$. 


\section{Conclusions}

In the earlier reasonings concerning the way of drawing conclusions about the behavior of the stochastic technological processes, the values of a few initial moments were used. It turns out that in many cases it is possible to find approximate distributions of these processes on the basis of the measurement data. Knowing the approximate distribution of a given process we have almost complete knowledge of its behavior. Owing to that, it is much easier for us to detect possible irregularities in the technological process.

\section{Acknowledgment}

This work has been financed by the Polish Ministry of Science and Higher Education, research project No. N N501 180636.

\section{References}

[1] M. Jabłoński, A. Ozga, Mechanics 25, 156 (2006).

[2] M. Jabłoński, A. Ozga, Archives of Acoustics 31, 373 (2006).

[3] H. Hurwitz, M. Kac, Annals of Mathematical Statistics, Vol. 15, 2, p. 173 (1944).

[4] R. Iwankiewicz, S. Nielsen, J. Sound Vib. 156, 407 (1992).
[5] R. Iwankiewicz, in: Proceedings of the 10th IFIP, WIG 7.5 Working Conference on Reliability and Optimization of Structural Systems, 25-27 March 2002, Kansai University, Osaka, Japan. Eds. 2003, p. 103.

[6] R. Iwankiewicz, Euromech 413, Stochastic Dynamics of Non-linear Mechanical Systems, 12-14 June 2000, Palermo, Italy in Meccanica, 37, No. 1, p. 12.

[7] A. Khintchine, Bull. Acad. Sci URSS, Ser. Math. 3, (1938).

[8] S.O. Rice, Mathematical analysis of random noise I, Bell. System Technical Journal 23, 1 (1944).

[9] J.B. Roberts, J. Sound Vib. 2, 336 (1965).

[10] J.B. Roberts, J. Sound Vib. 2, 375 (1965).

[11] J.B. Roberts, J. Sound Vib. 24, 23 (1972).

[12] J.B. Roberts, J. Sound Vib. 28, 93 (1973).

[13] J.B. Roberts, P.D. Spanos, Int. J. Non-Linear Mech. 21, 111 (1986).

[14] E.N. Rowland, Mathematical Proceedings of the Cambridge Philosophical Society 32, 580 (1936).

[15] L. Takác, Acta Mathematica Hungarica 5, 203 (1954).

[16] S. Kasprzyk, Dynamics of continuous systems, Wydawnictwa AGH, Cracow 1994.

[17] A. Plucińska, E. Pluciński, Probability, Wydawnictwa Naukowo-Techniczne, Warsaw 2000.

[18] K. Sobczyk, Stochastic Differential Equations, Wydawnictwa Naukowo-Techniczne, Warsaw 1996. 